IAU Colloquium 164: Radio Emission from Galactic and Extragalactic Compact Sources

ASP Conference Series, Vol. 144, 1998

J. A. Zensus, G. B. Taylor, \& J. M. Wrobel (eds.)

\title{
Position Angle of the HALCA Antenna Feed
}

\section{R. Kogan}

National Radio Astronomy Observatory, Socorro, NM 87801, U.S.A.

Abstract. Knowledge of the position angle of an antenna feed is very important for polarization observations. The issue had been developed for ground based antennas but has not been considered for a orbiting antennas. We provide a calculation of the position angle of the Japanese satellite VSOP/HALCA which was successfully launched in a space VLBI mission.

Let's define a right hand coordinate system fixed on the satellite $\left(X_{1}, Y_{1}, Z_{1}\right)$. Axis $Z_{1}$ points towards the source being observed and $Y_{1}$ is the axis about which the solar panels can be rotated. The solar panels are pointed at the Sun by rotation of the panels about axis $Y_{1}$ and rotation of the whole satellite about axis $Z_{1}$. Projection of the vector directed to the Sun on the antenna aperture is fixed in the coordinate system $\left(X_{1}, Y_{1}, Z_{1}\right)$ for all sources observed and for all times. This important statement allows us to determine the feed position angle relative to north by the formula:

$$
P A={\overrightarrow{s_{a}}}_{\vec{n}}+P A_{0}
$$

where $\vec{s}_{a}$ is the projection of the vector in the Sun direction on the antenna aperture;

$\vec{n}_{a}$ is the projection of the vector in the north direction on the antenna aperture;

$\vec{s}_{a} \cdot \vec{n}_{a}$ is the angle between vectors $\vec{s}_{a}$ and $\vec{n}_{a}$;

$P A_{\circ}$ is the angle in the antenna aperture between the reference vector $\vec{s}_{a}$ and major axis of the feed polarization ellipse;

Vector $\vec{s}_{a}$ can be determined from the following formula:

$$
\vec{s}_{a}=\vec{s}-\vec{e}(\vec{e} \cdot \vec{s})
$$

where $\vec{s}$ is the unit vector in the Sun direction;

$\vec{e}$ is the unit vector in the source direction;

$(\vec{e} \cdot \vec{n})$ is the scalar product of the vectors.

Vectors $\vec{s}$ and $\vec{e}$ have the following form in the equatorial coordinate system:

$$
\begin{aligned}
& \vec{s}=\left\{\cos \left(\alpha_{s}\right) \cos \left(\delta_{s}\right), \sin \left(\alpha_{s}\right) \cos \left(\delta_{s}\right), \sin \left(\delta_{s}\right)\right\} \\
& \vec{e}=\{\cos (\alpha) \cos (\delta), \sin (\alpha) \cos (\delta), \sin (\delta)\}
\end{aligned}
$$

where $\alpha_{s}, \delta_{s}$ are right ascension and declination of the Sun

$\alpha, \delta$ are right ascension and declination of the source

To find the angle $\vec{s}_{a} \cdot \vec{n}_{a}$ we need to determine the direction to the north and east in the aperture. Having found the projection of the reference vector $\vec{s}_{a}$ in these directions, we will be able to find both the absolute value of the angle 
$\vec{s}_{a} \cdot \vec{n}_{a}$ and its sign. The unit vectors $\vec{v}$ and $\vec{u}$ are known and correspond to the north and east direction respectively:

$$
\begin{aligned}
& \vec{v}=\{-\cos (\alpha) \sin (\delta),-\sin (\alpha) \sin (\delta), \cos (\delta)\} \\
& \vec{u}=\{-\sin (\alpha), \cos (\alpha), 0\}
\end{aligned}
$$

Using equations $(2,3,4,4)$ we can find the expression for the required projections:

$$
\begin{aligned}
\left(\vec{s}_{a} \cdot \vec{n}_{a}\right)=\left(\vec{s}_{a} \cdot \vec{v}\right) & =(\vec{s} \cdot \vec{v})=-\cos \left(\delta_{s}\right) \sin (\delta) \cos \left(\alpha_{s}-\alpha\right)+\sin \left(\delta_{s}\right) \cos (\delta) \\
\left(\vec{s}_{a} \cdot \vec{u}\right) & =(\vec{s} \cdot \vec{u})=\cos \left(\delta_{s}\right) \sin \left(\alpha_{s}-\alpha\right)
\end{aligned}
$$

Now finally the position angle measured from north to east can be found using the following expression:

$$
P A=\arctan \frac{\cos \left(\delta_{s}\right) \sin \left(\alpha_{s}-\alpha\right)}{-\cos \left(\delta_{s}\right) \sin (\delta) \cos \left(\alpha_{s}-\alpha\right)+\sin \left(\delta_{s}\right) \cos (\delta)}+P A_{\circ}
$$

Using equations (6), we have evaluated the position angle of the HALCA antenna feed for different source positions. For the ground-based antennas everything repeats over 24 hours and for sources with different right ascension shifted in time by the right ascensions difference. The situation is different for orbiting VLBI antennas. First of all, the typical time period is not 1 day but 1 year. Secondly nothing is repeatable for different right ascensions. That is why we need to provide the calculation for a range of both right ascension and declination. We can limit the right ascension range to $\left(0,180^{\circ}\right)$ because for the other half of the range the solution is repeated with opposite sign of the declination. The time range can be limited to a half year because the Sun's right ascension and declination for the second half of the year differs by $180^{\circ}$ and sign respectively. Such a difference provides the $180^{\circ}$ position angle change which is not important as discussed above. There are several special cases:

The source is located at the ecliptic plane.

We can put the axis $Y_{1}$ (axis of rotation of the solar panels) perpendicular to the ecliptic plane. HALCA can point the solar panels towards the Sun by rotation of the panels about the axis $Y_{1}$ without rotation of the satellite about the antenna axis $Z_{1}$. So the position angle is constant (the constant is between $66.4^{\circ}$ and $90^{\circ}$ ) throughout the year.

The Sun direction is close to the source (or to the opposite) direction.

In this case the pointing conditions to the both Sun and the source can be satisfied for any rotation of the satellite about the antenna axis. So the position angle is ambiguous.

Acknowledgments. The National Radio Astronomy Observatory is a facility of the NSF, operated under a cooperative agreement by Associated Universities, Inc. 